\title{
A Novel SPINK5 Gene Mutation Associated with Netherton Syndrome in an Omani Patient
}

"Nishath Hamza, ${ }^{1}$ Nashat Al Sukaiti, ${ }^{2}$ Khwater A.M. Ahmed, ${ }^{2}$ Rosa Romano, ${ }^{3}$ Uday A. Gokhale, ${ }^{4}$ Qiang Pan-Hammarström ${ }^{5}$

ABSTRACT: Netherton syndrome (NS) is an autosomal recessive primary immunodeficiency. It is characterised by substantial skin barrier defects and is often misdiagnosed as severe atopic dermatitis or hyper-immunoglobulin E syndrome. Although more than $80 \mathrm{NS}$-associated pathogenic mutations in the serine peptidase inhibitor kazal type 5 (SPINK5) gene have been reported worldwide, only one has been reported in the Arab population to date. We report the case of a novel association between the c.1887+1G>A mutation in the SPINK5 gene and NS in an Omani-Arab patient born in 2014 who was managed at a paediatric immunology clinic in Muscat, Oman. Accurate genetic diagnosis facilitated tailored clinical management of the index patient and enabled the provision of genetic counselling and offering of future reproductive options to the individuals related to the index patient.

Keywords: Netherton Syndrome; Primary Immunodeficiency Disease; Serine Peptidase Inhibitor Kazal Type 5; Congenital Ichthyosiform Erythroderma; Genetics; Case report; Oman.

$\mathrm{N}$ ETHERTON SYNDROME (NS) IS DEFINED AS an autosomal recessive primary immunodeficiency, which is characterised by a substantial skin barrier defect leading to ichthyosis being present at birth with erythroderma, which subsequently evolves into ichthyosis linearis circumflexa. ${ }^{1} \mathrm{NS}$ is caused by mutations in the serine peptidase inhibitor kazal type 5 (SPINK5) gene, which encodes the serine protease inhibitor lymphoepithelial Kazal-type inhibitor 1 (LEKTI) protein. NS patients exhibit severe atopic diathesis and a predisposition to allergies, asthma and eczema, with hypereosinophilia, elevated serum immunoglobulin (Ig)E and trichorrhexis invaginata (hair-shaft abnormality which leads to bamboo-like hair). ${ }^{2}$ Owing to its dermatological manifestations, NS is often misdiagnosed as severe atopic dermatitis or hyper-IgE syndrome. ${ }^{3,4}$ In NS patients, early genetic testing and diagnosis can prove critical to the provision of correct clinical management and decreased mortality.

To date, more than 80 NS-associated pathogenic mutations in the SPINK5 gene have been reported worldwide. ${ }^{5}$ While there have been reports of NS patients from the Middle East, to the best of the authors' knowledge, only one NS-related mutation has been reported so far among the Arab population. ${ }^{6}$ The current case report presents the case of a novel NSassociated mutation in an Omani-Arab patient with a family history of multiple affected individuals.

\section{Case Report}

The index patient was a male infant born in 2014, who presented to a paediatric immunology clinic in Muscat, Oman at 34 days of age. He was born to consanguineous parents and was affected with generalised erythematous desquamative skin rash from birth. At four months of age, the patient had normal IgG, IgA and IgM levels. Moreover, repeated immunepanel testing indicated normal complement C3, C4 and total haemolytic complement levels. In addition, he had a normal lymphocyte count, mildly elevated eosinophil and normal IgE, which later rose to $>1000$ IU/L on follow-up [Table 1]. Immunophenotyping indicated $27 \%$ of human leukocyte antigen (HLA)-DR isotype positive cells within the lymphocyte subset and the patient was reactive for antibody-specific response to hepatitis $B$ vaccine. The patient suffered recurrent infections from the age of one month, which increased in frequency and worsened in severity over time. Cultures from his blood, ears, cerebrospinal fluid and eyes showed colonisation by methicillin-resistant Staphylococcus aureus. Until the age of two years, he required peripheral red blood cell transfusion six times. At 27 months of age, the patient displayed failure to thrive, global developmental delay, severe anaemia and food allergy, indicated by high ImmunoCAP and radioallergosorbent test values of cow's milk and wheat [Table 1].

An older deceased male sibling had also presented with a skin condition similar to the index patient. This sibling was born by spontaneous vaginal delivery at home and was rushed to the nearest hospital with delay in crying, cyanosis and distress. He was intubated and ventilated for approximately four weeks in the new-born intensive care unit (NICU), where he was diagnosed by a dermatologist to have non-bullous

${ }^{1}$ National Genetic Center and Departments of ${ }^{2}$ Pediatric Allergy \& Clinical Immunology and ${ }^{4}$ Histopathology, Royal Hospital, Muscat, Oman; ${ }^{3}$ Division of Pediatric Stem Cell Transplantation \& Regenerative Medicine, Stanford University, Stanford, USA; ${ }^{5}$ Department of Biosciences E Nutrition, Karolinska University Hospital, Stockholm, Sweden

*Corresponding Author e-mail: nishahamz@yahoo.co.uk 
Table 1: Immunological investigations of the index patient

\begin{tabular}{|c|c|c|}
\hline Parameter & Result & $\begin{array}{l}\text { Reference } \\
\text { range }\end{array}$ \\
\hline \multicolumn{3}{|c|}{ Investigations at the age of four months } \\
\hline \multicolumn{3}{|l|}{ Lymphocyte count in $\times 10^{9} / \mathrm{L}$} \\
\hline Total lymphocytes in $\times 10^{9} / \mathrm{L}$ & 7.91 & $3.6-8.8$ \\
\hline T-cells $(\mathrm{CD} 3+)$ in $\times 10^{9} / \mathrm{L}$ & 5.19 & $2.3-6.5$ \\
\hline B-cells $(\mathrm{CD} 19+)$ in $\times 10^{9} / \mathrm{L}$ & 1.32 & $0.5-1.5$ \\
\hline $\begin{array}{l}\text { T-helper cells }(\mathrm{CD} 3+/ \mathrm{CD} 4+) \\
\text { in } \times 10^{9} / \mathrm{L}\end{array}$ & 2.63 & $1.7-4.6$ \\
\hline $\begin{array}{l}\text { T-cytotoxic cells (CD3+/ } \\
\text { CD } 8+) \text { in } \times 10^{9} / \mathrm{L}\end{array}$ & 1.46 & $0.7-3.5$ \\
\hline $\mathrm{CD} 4: \mathrm{CD} 8$ ratio in $\times 10^{9} / \mathrm{L}$ & 1.79 & $1.2-3.5$ \\
\hline $\begin{array}{l}\text { T-helper CD4+ count in } \\
\text { cells/uL }\end{array}$ & 2,630 & \\
\hline Eosinophils in percentage & 2.6 & $0.1-0.8$ \\
\hline \multicolumn{3}{|l|}{ Serum Ig } \\
\hline IgE in IU/L & 193 & $0-15$ \\
\hline IgG in $g / L$ & 6.0 & $2.05-9.5$ \\
\hline IgA in $g / L$ & 0.89 & $0.08-0.91$ \\
\hline IgM in $g / L$ & 0.58 & $0.17-1.5$ \\
\hline \multicolumn{3}{|l|}{ Serum complement levels } \\
\hline $\mathrm{C} 3$ in $\mathrm{mg} / \mathrm{L}$ & 968 & $820-1,850$ \\
\hline $\mathrm{C} 4$ in $\mathrm{mg} / \mathrm{L}$ & 283 & $150-530$ \\
\hline $\mathrm{CH} 50 \mathrm{in} \%$ & 71 & $70-140$ \\
\hline \multicolumn{3}{|c|}{ Investigations at the age of two years } \\
\hline Serum IgE in IU/L & 1526 & $0-60$ \\
\hline \multicolumn{3}{|l|}{ Allergy testing for specific IgE* } \\
\hline Cow's milk in kU/L & 20.60 & - \\
\hline Wheat in $\mathrm{kU} / \mathrm{L}$ & 9.25 & \\
\hline
\end{tabular}

congenital ichthyosiform erythroderma. During his stay in the NICU, he developed sepsis with coagulasenegative staphylococci and candida. By 70 days of age, the sibling had rapidly developed septic shock and died while he was admitted. Upon probing further, the parents of the index patient revealed a history of infant deaths of the index patient's paternal cousins, who had died of sepsis before the age of one and had received diagnoses of atopic dermatitis [Figure 1].

Given this family history and the propensity for recurrent infections, the index patient was suspected of having a primary immunodeficiency. Later, hair biopsy and histopathology evaluations of the index patient at five months of age using Haemotoxylin showed psoriasiform

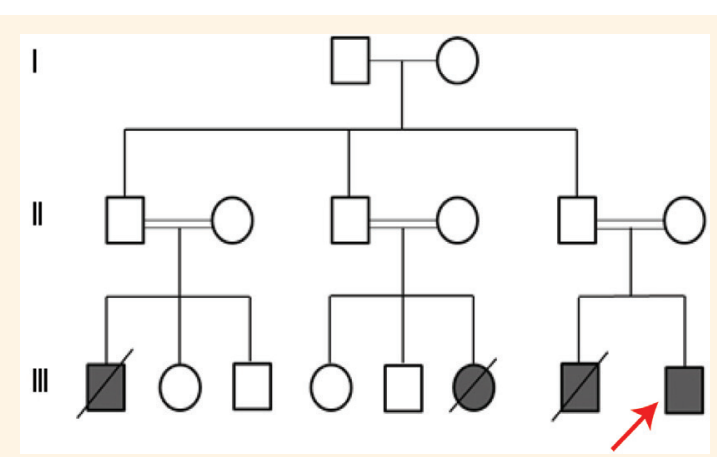

Figure 1: Family pedigree of the index patient. A sibling of the index patient (red arrow) was affected and had previously died of sepsis. Two paternal cousins who presented with features similar to the index patient also died of sepsis before the age of one year.

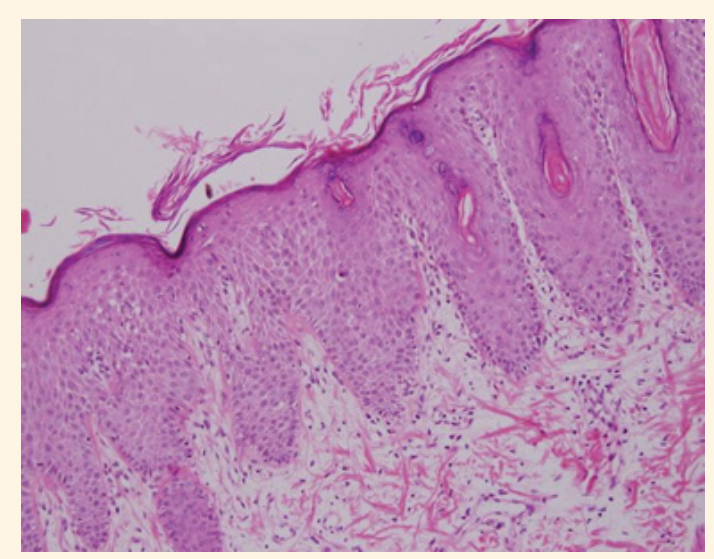

Figure 2: Haemotoxylin and eosin stain of a skin biopsy of the index patient at $\times 10$ magnification showing psoriasiform acanthosis spongiosis, mild lymphocytic exocytosis with occasional dyskeratotic cells.

acanthosis spongiosis and mild lymphocytic exocytosis with occasional dyskeratotic cells, which indicated a clinical diagnosis of NS [Figure 2].

Whole exome sequencing (WES) of the index patient's DNA in 2016 revealed a homozygous mutation c.1887+1G>A (NM_001127699.2; hg19:Chr5:147492498) in intron 20 of the SPINK5 gene associated with autosomal-recessive NS. This mutation affects the highly conserved consensus donor splice site at the exon 20-intron 20 junction. This variant is present in the dbSNP database (rs1042707088; National Center for Biotechnology Information, Bethesda, Maryland, USA) and was detected only twice in heterozygous form within a human longevity project from 2016 but was never associated with any clinical presentation. Sanger sequencing technique further validated the WES result and confirmed the segregation of the c.1887+1G>A mutation in heterozygous form in each parent. The c.1887+1G>A variant was initially classified as a variant of uncertain significance (VUS) due to the lack of population frequency data or functional evidence. However, the ever-increasing 

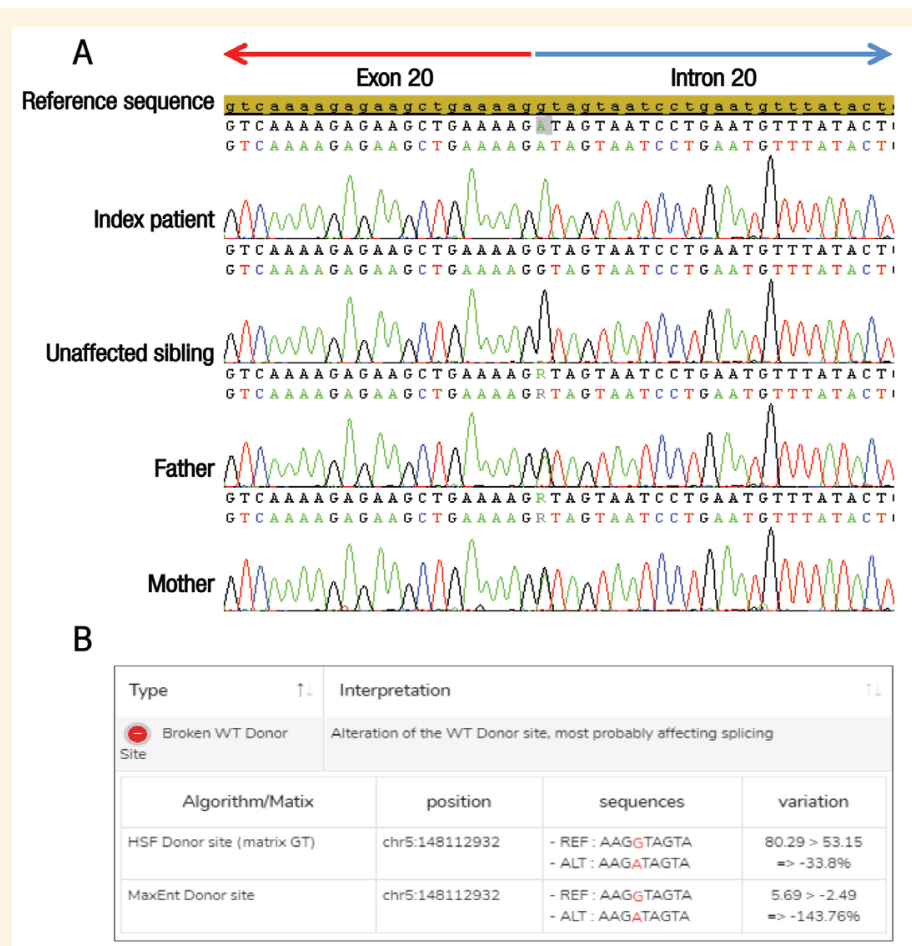

Figure 3: Outputs showing evidence for pathogenicity of the c.1888+1G>A mutation. A: Family segregation analysis using Sanger sequencing data of the exon 20-intron 20 junction shows the c.1888+1G>A mutation as homozygous in the index patient (asterisk) and as heterozygous ( $R$ indicates G/A transition) in the parents of the index patient. The unaffected sibling of the index patient does not carry the mutated allele. B: Results from the in silico analysis using the Human Splicing Finder suite (GENOMNIS) indicates that the c.1888+1G>A mutation probably affects splicing at the exon 20 -intron 20 junction.

volume of exome sequencing data compiled since 2016 at the two laboratories participating in this study and the absence of this variant in online population databases enabled the authors to ascertain that this mutation was indeed rare and novel with respect to NS. Moreover, an unaffected sibling of the index patient born in 2020 was also screened and found to carry only normal SPINK5 alleles [Figure 3A].

The potential impact of the c.1887+1G>A mutation was further analysed using the in silico Human Splicing Finder (HSF) suite (GENOMNIS) which evaluates a candidate's splice site mutations using both the HSF matrix and the MaxEntScan matrix (Burge Lab, Massachusetts Institute of Technology, Massachusetts, USA) ${ }^{8,9}$ Both matrices predicted the c.1887+1G>A mutation observed in the index patient to 'probably affect splicing' [Figure 3B].

Interestingly, another mutation, c.1888-1G>A (hg19:Chr5:147493924), affecting the same intron 20 at the opposite end (i.e. the acceptor splice site at the intron 20-exon 21 junction) had already been reported as being pathogenic in NS patients. ${ }^{9,10}$ It was observed that the previously reported c.1888-1G>A mutation and the c.1887+1G>A mutation reported here scored 3.83 and 3.85 , respectively, on measurements of evolutionary conservation by two algorithms-
PhastCons and PhyloP-from the Phylogenetic Analysis with Space/Time models (PHAST) package (Cold Spring Harbor Laboratory, New York, USA) generated using the bioinformatic tools in the University of California Santa Cruz comparative genomics alignment pipeline. ${ }^{11}$ This indicated that both mutations (separated by $>1.4 \mathrm{kbp}$ ) were located at equally and highly conserved splice sites at opposite ends of intron 20 of the SPINK5 coding sequence. Given all of the above evidence, the c.1887+1G>A mutation was hence re-classified as 'pathogenic' and predicted to result in aberrant LEKTI protein expression in homozygous patients.

An accurate genetic diagnosis enabled a tailored clinical strategy whereby intravenous (IV) Ig therapy $(0.4 \mathrm{~g} / \mathrm{kg} / \mathrm{month})$ was administered and led to decreased inflammation and itching of the skin; it also led to thicker hair with reduced hair-shaft breakage and a healthier scalp. At the time of the last clinical follow-up, the index patient was at 33 months of age. His skin condition had vastly improved, albeit with some generalised persistent redness. While he still suffered from developmental delay, he had started to reach milestones such as being able to sit without support. 
Informed consent for testing and publication of anonymised data was collected from all patients/ guardians involved in this study; appropriate ethical standards were employed in all procedures.

\section{Discussion}

This case report presents a novel NS-associated c. $1887+1 G>$ A mutation in the SPINK5 gene. Although this mutation was observed in the index patient in 2016, it was initially classified as a VUS. However, with access to growing data from subsequent exome sequencing studies over the past years, it is evident that the c.1887+1G>A mutation was indeed rare and likely to be pathogenic in association with NS in the index patient. Given that more than $50 \%$ of marriages in Oman are reported to be consanguineous unions, the prospect of this being a founder mutation cannot be ignored.12 Hence, the detection of novel variants in rare disorders such as NS facilitates a targeted screening approach for quicker diagnosis in future cases.

SPINK5 gene mutations can result in LEKT1 deficiency. LEKTI has been shown to inhibit several members of the serine protease family such as kallikrein related peptidase (KLK)5, KLK7 and KLK14 as well as trypsin, plasmin, subtilisin $\mathrm{A}$, cathepsin $\mathrm{G}$ and human neutrophil elastase. ${ }^{13}$ Unhindered functioning of these serine proteases can trigger a cascade of events, whereby increased proteolysis destroys the stratum corneum integrity, affects lipid barrier maintenance and exacerbates desquamation of the epidermis. ${ }^{13}$ These effects conceivably compromise the antimicrobial-barrier function of the skin in NS patients, thereby leading to colonisation of lesional and non-lesional skin by S. aureus-a presentation reported in both the index patient and his deceased sibling.

Unearthing the genetic aetiology of the index patient's symptoms was crucial in providing correct clinical management and likely helped avoid the severe complications observed in the index patient's older sibling and deceased paternal cousins. While there are multiple treatment modalities targeting skin manifestations and immune defects, symptomatic treatment with allergy control medication, moisturisers, steroid creams and antibiotics has limited effect on NS patients. However, IVIg replacement therapy has been reported to be quite effective in NS patients as was seen in the current patient. ${ }^{14}$

Retrospective evaluation of the case histories of the previously deceased paternal cousins of the index patient indicated that they had likely been affected with NS as well. This was unsurprising as all the affected offspring were born of consanguineous unions. Therefore, mutation carrier screening was offered to other individuals within the extended family, especially to provide pre-marital counselling for unmarried heterozygous carriers who intended to seek consanguineous unions. The family members with previously deceased offspring were also counselled to educate them about alternative reproductive options to avoid the recurrence of affected offspring. In Oman, prenatal genetic screening combined with assisted reproduction is usually offered as a safe and culturally acceptable option to such affected families.

\section{Conclusion}

NS is a rare and severe disease, which can be lifethreatening in infants without accurate diagnosis and tailored management. Genetic diagnosis of NS patients can have significant implications for their correct and effective clinical management using IVIg, which helps in reduction of infections as well as ameliorates skin and hair anomalies. Moreover, genetic testing for patients with rare diseases such as NS also facilitates the provision of genetic counselling and selection of future reproductive options provided to affected families, particularly in societies that are highly consanguineous and opposed to abortion.

\section{AUTHORS' CONTRIBUTION}

$\mathrm{NH}$ organised the study, analysed the data and drafted the manuscript. NS provided clinical supervision, while NS and KA collected clinical data and reviewed the manuscript. RR and UG analysed the patient data. $\mathrm{QPH}$ reviewed the data and manuscript. All authors approved the final version of the manuscript.

\section{References}

1. Sun JD, Linden KG. Netherton syndrome: A case report and review of the literature. Int J Dermatol 2006; 45:693-7. https:// doi.org/10.1111/j.1365-4632.2005.02637.x.

2. Hannula-Jouppi K, Laasanen SL, Ilander M, Furio L, Tuomiranta M, Marttila R, et al. Intrafamily and interfamilial phenotype variation and immature immunity in patients with Netherton syndrome and Finnish SPINK5 founder mutation. JAMA Dermatol 2016; 152:435-42. https://doi.org/10.1001/jamader matol.2015.5827.

3. Leung AKC, Barankin B, Leong KF. An 8-year-old child with delayed diagnosis of Netherton syndrome. Case Rep Pediatr 2018; 2018:9434916. https://doi.org/10.1155/2018/9434916.

4. Özyurt K, Atasoy M, Ertaş R, Ulaş Y, Akkuş MR, Kiraz A, et al. Netherton syndrome previously misdiagnosed as hyper IgE syndrome caused by a probable mutation in SPINK5 C. Turk J Pediatr 2019; 61:604-7. https://doi.org/10.24953/ turkjped.2019.04.020. 
5. Sarri CA, Roussaki-Schulze A, Vasilopoulos Y, Zafiriou E, Patsatsi A, Stamatis C, et al. Netherton syndrome: A genotypephenotype review. Mol Diagn Ther 2017; 21:137-52. https:// doi.org/10.1007/s40291-016-0243-y.

6. Nevet MJ, Indelman M, Ben-Ari J, Bergman R. A case of Netherton syndrome with intestinal atresia, a novel SPINK5 mutation, and a fatal course. Int J Dermatol 2017; 56:1055-7. https://doi.org/10.1111/ijd.13730.

7. Desmet FO, Hamroun D, Lalande M, Collod-Béroud G, Claustres M, Béroud C. Human Splicing Finder: An online bioinformatics tool to predict splicing signals. Nucleic Acids Res 2009; 37:e67. https://doi.org/10.1093/nar/gkp215.

8. Yeo G, Burge CB. Maximum entropy modeling of short sequence motifs with applications to RNA splicing signals. J Comput Biol 2004; 11:377-94. https://doi.org/10.1089/1066527041410418.

9. Chavanas S, Bodemer C, Rochat A, Hamel-Teillac D, Ali $\mathrm{M}$, Irvine $\mathrm{AD}$, et al. Mutations in SPINK5, encoding a serine protease inhibitor, cause Netherton syndrome. Nat Genet 2000; 25:141-2. https://doi.org/10.1038/75977.
10. Descargues P, Deraison C, Prost C, Fraitag S, MazereeuwHautier J, D'Alessio M, et al. Corneodesmosomal cadherins are preferential targets of stratum corneum trypsin- and chymotrypsin-like hyperactivity in Netherton syndrome. J Invest Dermatol 2006; 126:1622-32. https://doi.org/10.1038/ sj.jid. 5700284 .

11. Kent WJ, Sugnet CW, Furey TS, Roskin KM, Pringle TH, Zahler AM, et al. The human genome browser at UCSC. Genome Res 2002; 12:996-1006. https://doi.org/10.1101/gr.229102.

12. Rajab A, Hamza N, Al Harasi S, Al Lawati F, Gibbons U, Al Alawi I, et al. Repository of mutations from Oman: The entry point to a national mutation database. F1000Res 2015; 4:891. https://doi.org/10.12688/f1000research.6938.1.

13. Furio L, Hovnanian A. Netherton syndrome: Defective kallikrein inhibition in the skin leads to skin inflammation and allergy. Biol Chem 2014; 395:945-58. https://doi.org/10.1515/ hsz-2014-0137.

14. Renner ED, Hartl D, Rylaarsdam S, Young ML, MonacoShawver L, Kleiner G, et al. Comèl-Netherton syndrome defined as primary immunodeficiency. J Allergy Clin Immunol 2009; 124:536-43. https://doi.org/10.1016/j.jaci.2009.06.009. 